Original Article

\title{
Association of Perioperative Redox Balance on Long-Term Outcome in Patients Undergoing Lung Resection
}

\author{
Osamu Araki, MD, ${ }^{1}$ Yuji Matsumura, MD, PhD, ${ }^{2}$ Takashi Inoue, MD,${ }^{1}$ Yoko Karube, MD, PhD, ${ }^{1}$ \\ Sumiko Maeda, MD, PhD, ${ }^{1}$ Satoru Kobayashi, MD, PhD,${ }^{1}$ and Masayuki Chida, MD, $\mathrm{PhD}{ }^{1}$
}

\begin{abstract}
Purpose: We examined whether redox balance during the perioperative period is associated with long-term survival of patients after undergoing lung resection.

Methods: Patients who underwent surgery for lung cancer from January to June 2013 at our institution were investigated. Serum was collected during the operation, and on postoperative day (POD) 3 and 7, and the levels of derivatives of reactive oxygen metabolites (d-ROMs) and biologic antioxidant potential (BAP) were measured.

Results: In all, 21 patients ( $69 \pm 7$ years old) were enrolled, of whom 9 underwent videoassisted thoracoscopic surgery. Comorbidities in the patients included chronic obstructive pulmonary disease in eight and idiopathic pulmonary fibrosis in five. d-ROM values on POD 3 and 7 were significantly increased as compared to those obtained during the operation $(p<0.001)$, whereas BAP did not change after surgery. Patients with a value below a d-ROM cutoff value of 327 during the operation showed significantly superior 3-year overall survival as compared to those with a value above the cutoff $(87.5 \%$ vs. $20.0 \%$, p $<$ 0.001).

Conclusion: In the present patients, surgical stress caused an increase in d-ROM during the postoperative course. The $\mathrm{d}-\mathrm{ROM}$ value obtained during the operation was correlated with long-term survival following resection for lung cancer.
\end{abstract}

Keywords: lung cancer, redox balance, oxidative stress, reactive oxygen species

\section{Introduction}

Surgical stress can induce a cytokine storm and systemic inflammatory response syndrome, and also has effects on redox balance during the postoperative course.

${ }^{1}$ Department of General Thoracic Surgery, Dokkyo Medical University, Mibu, Tochigi, Japan

${ }^{2}$ Department of Cardiothoracic Surgery, Dokkyo Koshigaya Hospital, Koshigaya, Saitama, Japan

Received: July 26, 2017; Accepted: August 24, 2017

Corresponding author: Yuji Matsumura, MD, PhD. Department of Cardiothoracic Surgery, Dokkyo Koshigaya Hospital, 2-1-50 Minamikoshigaya, Koshigaya, Saitama 343-8555, Japan

Email: yuji-m@dokkyomed.ac.jp

(C)2018 The Editorial Committee of Annals of Thoracic and Cardiovascular Surgery. All rights reserved.
Oxidative stress can be defined as an imbalance between the pro-oxidant and antioxidant potential of cells, which results from an overproduction of reactive oxygen species (ROS) or insufficient detoxification of ROS by antioxidants, or those in combination. ${ }^{1)}$ It is widely known that ROS function as key metabolites that can impair biologic processes, resulting in various serious conditions, including cardiovascular disease, diabetes mellitus, neurologic disorders, and cancer. ${ }^{2-5)}$ However, whether inflammatory status, especially redox balance, during the perioperative period has a relationship with long-term outcome following surgery for lung cancer remains unclear. In this study, we aimed to determine whether redox balance during the perioperative period in patients after undergoing lung resection is associated with long-term survival. 


\section{Subjects and Methods}

We investigated consecutively treated patients who underwent an anatomical lung resection greater than a segmentectomy for non-small-cell lung cancer from January to June 2013 at our institution. Each provided informed consent and the Ethical Committee of Dokkyo Medical University Hospital approved this study (\#24043). Overall survival and cause of death were analyzed in this cohort.

Serum was collected during the operation, as well as on postoperative day (POD) 3 and 7, and the levels of derivatives of reactive oxygen metabolites (d-ROMs) and biologic antioxidant potential (BAP) were measured using a free radical elective evaluator system (FREE Carpe Diem, Wismerll Co. Ltd., Tokyo, Japan). Briefly, $20-\mu \mathrm{L}$ serum samples were added to a $1-\mathrm{mL}$ assay mixture, and then the resulting solution was gently mixed and incubated for 1 minute at $37^{\circ} \mathrm{C}$. The optical density of the total level of hydroperoxides was measured at $505 \mathrm{~nm}$ using a spectrophotometer. The results are expressed in arbitrary units, namely Carratelli units (U.CARR), with 1 U.CARR corresponding to $0.08 \mathrm{mg} / 100 \mathrm{~mL} \mathrm{H}_{2} \mathrm{O}_{2}{ }^{6}{ }^{6}$ BAP was also measured at the same time, for which $10-\mu \mathrm{L}$ serum samples were added to a $1-\mathrm{mL}$ assay mixture, then the amount of trivalent iron in the solution deoxidized over a period of 5 minutes was measured in units of $\mu \mathrm{mol} / \mathrm{L}$. From those results, the d-ROM to BAP ratio (d-ROM/BAP) was calculated.

Routine laboratory measurements including serum C-reactive protein (CRP), albumin, and complete blood cell count were also performed on the day before the lung resection to examine nutrition and inflammatory status.

Statistical analysis was performed using Student's t-test for comparisons between two groups. Receiver operating characteristic (ROC) curve analysis was done to estimate optimal sensitivity and specificity for prediction of all causes of death according to the cutoff level of each variable. The Kaplan-Meier method was used for calculation of survival from death by any cause. Comparisons within each group were performed using a log-rank test. All calculations were done using the JMP statistical software package (SAS Institute Inc., Cary, NC, USA). Differences were considered significant at $\mathrm{p}<0.05$.

\section{Results}

In all, 21 patients were enrolled in this study and their characteristics are shown in Table $\mathbf{1}$.
Table 1 Patient characteristics $(n=21)$

\begin{tabular}{lc}
\hline Age, years (range) & 68 (49-77) \\
Gender & \\
Male & 17 \\
Female & 4 \\
Histology & \\
Ad & 11 \\
Sq & 6 \\
Others & 4 \\
Stage & \\
Stage I & 14 \\
Stage II & 3 \\
Stage III & 3 \\
Stage IV & 1 \\
Surgical procedure & \\
VATS & 9 \\
Open thoracotomy & 12 \\
Lobectomy & 20 \\
Pneumonectomy & 1 \\
Smoking index (range) & $1047(0-3000)$ \\
Lung comorbidities & \\
COPD & 7 \\
IP & 5 \\
Other comorbidities & \\
DM & 6 \\
HTN & 5 \\
HL & 3 \\
\end{tabular}

Ad: adenocarcinoma; Sq: squamous cell carcinoma; VATS: video-assisted thoracic surgery; COPD: chronic obstructive lung disease; IP: interstitial pneumonia; DM: diabetes mellitus; HTN: hypertension; HL: hyperlipidemia

\section{Perioperative changes in d-ROM, BAP, and $\mathrm{d}-\mathrm{ROM} / \mathrm{BAP}$ values}

As compared to the perioperative d-ROM value (299 \pm 89 ), those on POD 3 and 7 (431 \pm 70 and $470 \pm 60$, respectively) were significantly increased $(\mathrm{p}<0.01)$. In contrast, BAP was not significantly different after surgery $(2441 \pm 586,2600 \pm 545$, and $2681 \pm 515$, respectively) (Figs. 1A and 1B), whereas the values for d-ROM/BAP on POD 3 and $7(0.172 \pm 0.038$ and 0.183 \pm 0.045 , respectively) were significantly increased $(\mathrm{p}<0.01)$ as compared to the perioperative value $(0.128$ $\pm 0.041)$ (Fig. 1C). There were no significant differences in regard to these parameters between video-assisted thoracoscopic surgery and open thoracotomy cases at the observed time points.

\section{Relationship of d-ROM and d-ROM/BAP values with long-term outcome}

During the observation period, seven patients had cancer relapse, of whom six died (lung cancer recurrence in 

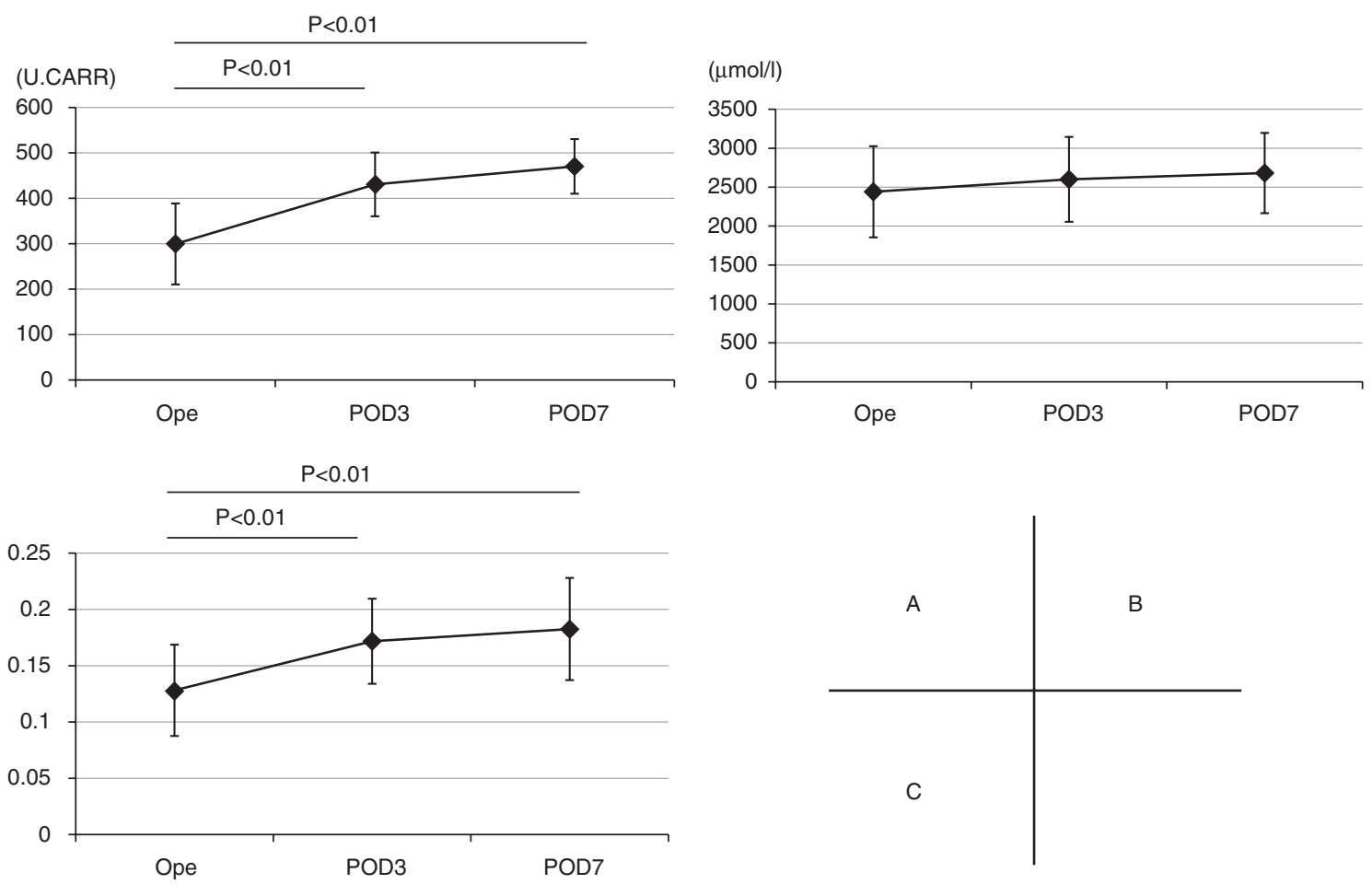

Fig. 1 (A) Perioperative changes in d-ROM, (B) BAP, and (C) d-ROM/BAP values. d-ROM: derivatives of reactive oxygen metabolite; BAP: biologic antioxidant potential; Ope: value obtained during operation; POD: postoperative day

three, pneumonia in two, and myocardial infarction in one patient). Overall survival was $71.4 \%$ after 3 years. ROC curve analysis revealed a d-ROM cutoff value of 327 during the operation, with values for sensitivity and specificity of 0.667 and 0.067 , respectively, and an area under the curve (AUC) value of 0.811. Patients with a d-ROM value $<327$ showed significantly superior 3-year survival as compared to those with a value $\geq 327$ (87.5\% vs. $20.0 \%$, p <0.001) (Fig. 2A). ROC curve analysis of d-ROM/BAP revealed a cutoff value of 0.122 during the operation (sensitivity: 0.833, specificity: 0.400), and an AUC value of 0.711. Patients with a d-ROM/BAP value $\geq 0.122$ showed inferior 3 -year survival as compared to those with a value $<0.122$ (90.0\% vs. $54.5 \%, \mathrm{p}=0.070$ ) (Fig. 2B). ROC curve analysis of $\mathrm{d}-\mathrm{ROM}$ POD3/POD0 values revealed a cutoff value of 1.78 (sensitivity: 0.500, specificity: 0.133), and an AUC value of 0.522. Patients with a d-ROM POD3/POD0 value $\geq 1.78$ had a 3 -year survival rate of $40 \%$ as compared to $80 \%$ for those with a value $<1.78(\mathrm{p}=0.102)$ (Fig. 3A). ROC curve analysis of d-ROM POD7/POD0 values revealed a cutoff value of 1.92 (sensitivity: 0.500, specificity: 0.067), and an AUC value of 0.511 . Patients with a d-ROM
POD7/POD0 value $\geq 1.92$ had a 3 -year survival rate of $25 \%$ as compared to $83 \%$ for those with a value $<1.92$ $(\mathrm{p}=0.029)$ (Fig. 3B).

\section{Inflammatory and nutritional prognostic factors}

Values for albumin, CRP, red cell deviation width (RDW), and neutrophil-to-lymphocyte ratio (NLR) in all patients were examined as inflammatory and nutritional prognostic factors. Since a cutoff value for d-ROM of 327 during the operation was most significant for indicating long-term survival, patients were divided into those with a d-ROM value $<327(n=16)$ and $\geq 327(n=5)$. The $d-R O M \geq 327$ group showed significantly higher values for CRP $(0.188+0.172$ vs. $1.69+2.74)$ and NLR $(1.96+0.75$ vs. $4.15+2.11)$ as compared to the d-ROM $<327$ group (Fig. 4).

\section{Discussion}

Our results showed that surgical stress increased the value for d-ROM, an index of oxidative stress, while it also resulted in deteriorated redox balance, as represented by d-ROM/BAP ratio, during the early period following a lung cancer resection procedure. The primary 

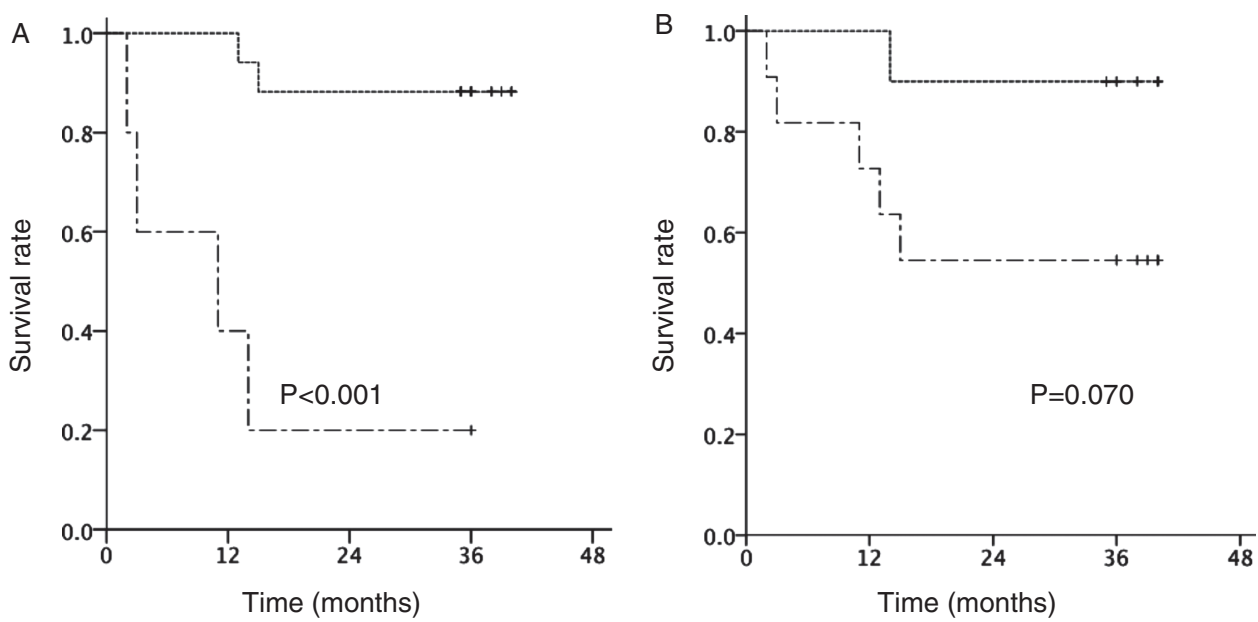

Fig. 2 Associations of d-ROM value and d-ROM/BAP ratio during operation with overall survival. (A) Overall survival curve of patients divided by d-ROM value during operation. Solid line: d-ROM $<327$, dotted line: $d-R O M \geq 327$. (B) Overall survival curve of patients divided by d-ROM/BAP ratio during operation. Solid line: d-ROM/BAP $<0.122$, dotted line: d-ROM/ $\mathrm{BAP} \geq 0.122$. d-ROM: derivatives of reactive oxygen metabolite; BAP: biologic antioxidant potential
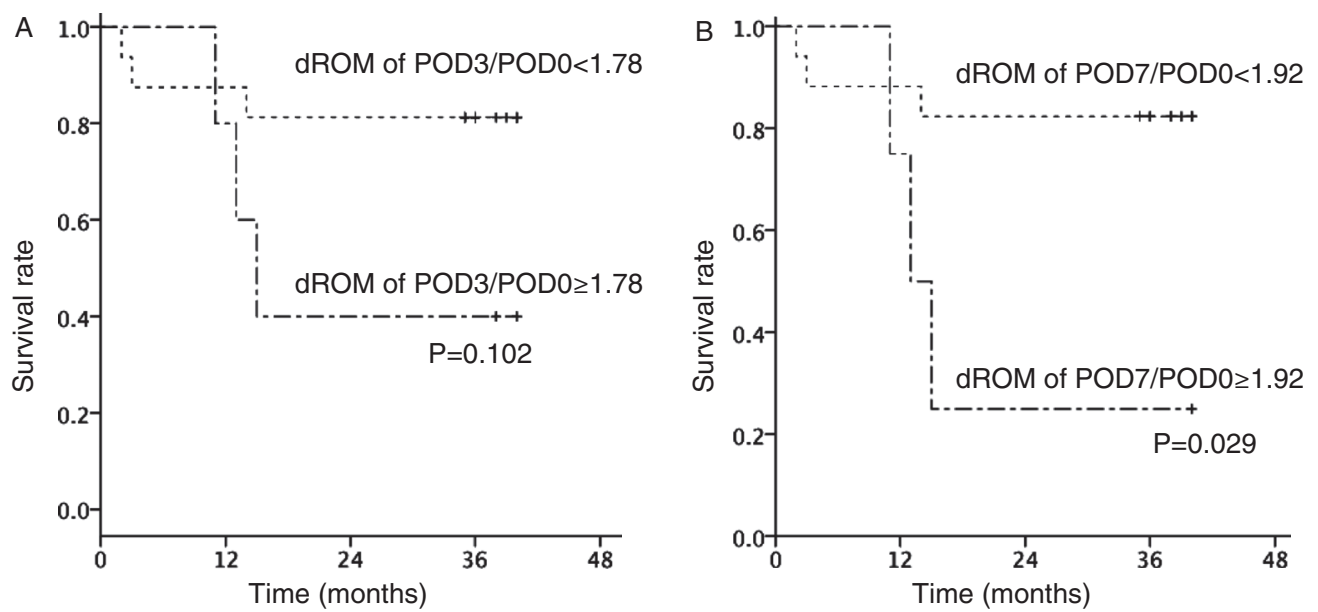

Fig. 3 Association of (A) POD3/POD0 and (B) POD7/POD0 d-ROM on overall survival. d-ROM: derivatives of reactive oxygen metabolite; POD: postoperative day

finding of this study is that d-ROM during surgery for lung cancer is most significantly associated with longterm survival as compared to the other parameters examined, regardless of cause of death after the operation. The group of patients with a d-ROM value during the operation less than the cutoff also had lower values for CRP and NLR, markers reported to be predictors of survival following lung cancer resection.

Surgical stress during the perioperative period influences redox balance in patients undergoing a cancer resection procedure. We found that d-ROM was significantly increased after surgery as compared to the perioperative value, which was the same for BAP, though the difference was not significant. As a result, the d-ROM/BAP ratio was also increased following surgery. Kanaoka et al. ${ }^{2)}$ reported an increase in ROMs including d-ROM following cardiovascular surgery. However, changes in BAP values during the perioperative period remain controversial, as both increases ${ }^{2)}$ and decreases ${ }^{7)}$ have been reported.

In the present study, we investigated whether these values were associated with long-term outcome in patients 

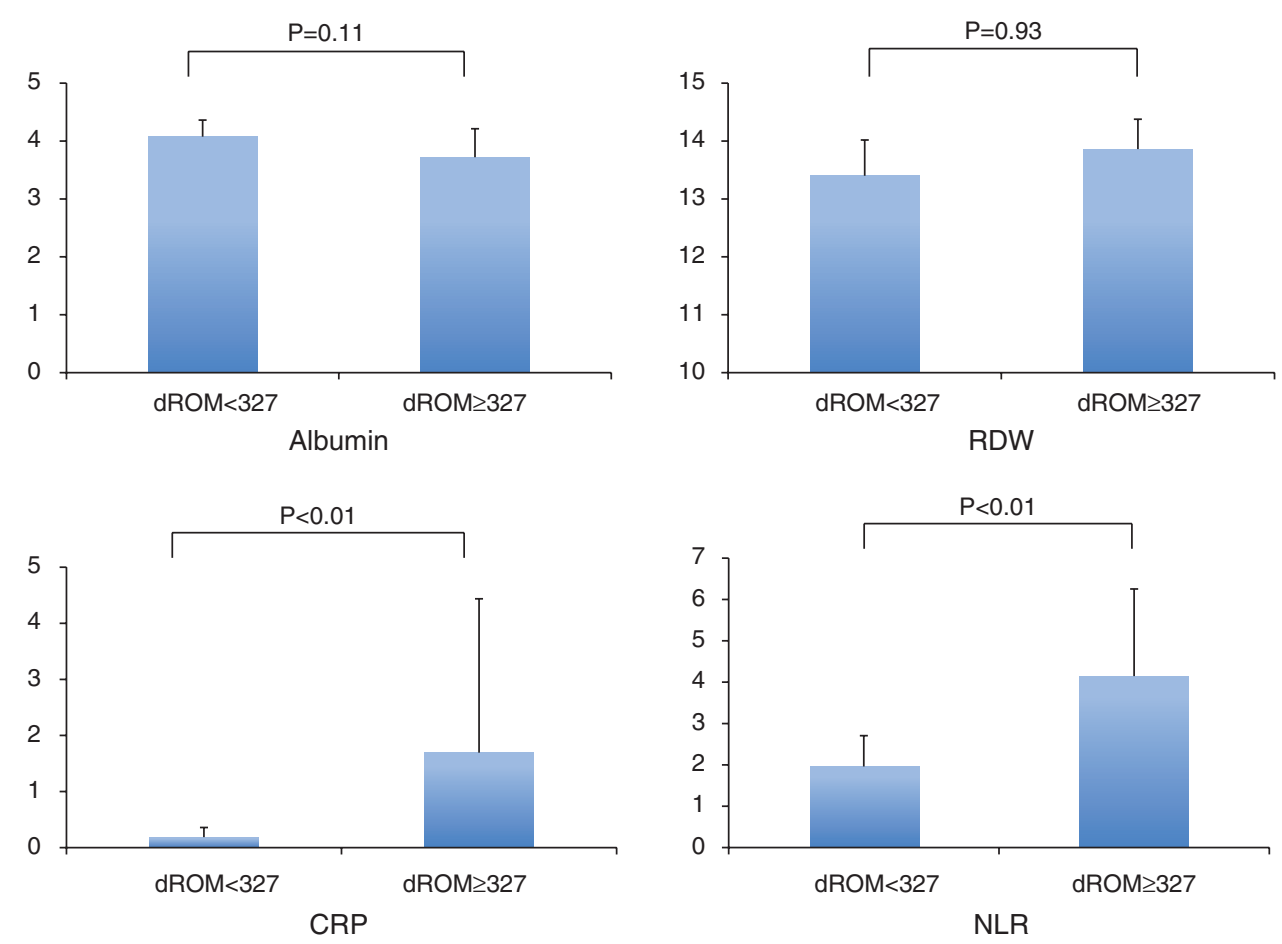

Fig. 4 Albumin, RDW, CRP, and NLR in association with d-ROM cutoff level. d-ROM: derivatives of reactive oxygen metabolite; RDW: red cell deviation width; CRP: C-reactive protein; NLR: neutrophil-to-lymphocyte ratio

who underwent resection surgery. We found that BAP did not show significant changes during the postoperative period; thus, we investigated the association of d-ROM with survival and analyzed d-ROM values obtained during operation, as well as on POD 3 and 7 , as well as the POD3/POD0 and POD7/POD0 ratios, and d-ROM/BAP ratio. Each of these showed a relationship with long-term survival following surgery. However, the only values to reach the level of significance were those for $\mathrm{d}-\mathrm{ROM}$ during the operation $(\mathrm{p}<0.001)$ and the POD7/POD0 d-ROM value $(\mathrm{p}=0.029)$, whereas $\mathrm{d}-\mathrm{ROM} / \mathrm{BAP}(\mathrm{p}=0.070)$ and POD3/POD0 d-ROM ( $\mathrm{p}=$ 0.102) were not significant. Furthermore, d-ROM on POD 3 and 7 did not show a correlation with survival (data not shown). Our results indicate that patients with elevated d-ROM during the operation, as well as during the near-term following surgery (POD7/POD0) have inferior long-term survival following a lung cancer resection procedure.

Our findings led us to question why d-ROM during and soon after surgery is related to long-term survival. In the present study, we focused on inflammatory and nutrition predictors (CRP, NLR, RDW, albumin), which have been shown to be correlated with outcome of patients with a malignant neoplasm. ${ }^{8-12)}$ Our patients with a higher d-ROM value during the operation also had elevated CRP and NLR before the operation, and their prognosis was predicted to be poor based on the preoperative inflammatory markers. Several comorbidities, such as smoking and diabetes mellitus, have been reported to influence redox balance. ${ }^{2-4,13)}$ Tsukioka and colleagues ${ }^{3,14)}$ also noted that preoperative d-ROM was correlated with nodal involvement, which affects prognosis following lung cancer resection. Thus, perioperative d-ROM may be a predictor of long-term survival following resection for lung cancer.

The present study is limited by its design as an institutional report and the low number of subjects investigated. Additional studies and accumulation of cases are necessary.

\section{Conclusion}

We found that surgical stress causes an increase in d-ROM during the early postoperative course. Furthermore, the $\mathrm{d}-\mathrm{ROM}$ value obtained during the operation was correlated with long-term survival of patients who underwent a lung cancer resection procedure. 


\section{Disclosure Statement}

None was declared.

\section{References}

1) Salzman R, Pácal L, Kaňková K, et al. High perioperative level of oxidative stress as a prognostic tool for identifying patients with a high risk of recurrence of head and neck squamous cell carcinoma. Int J Clin Oncol 2010; 15: 565-70.

2) Kanaoka Y, Inagaki E, Hamanaka S, et al. Analysis of reactive oxygen metabolites (ROMs) after cardiovascular surgery as a marker of oxidative stress. Acta Med Okayama 2010; 64: 323-30.

3) Tsukioka T, Nishiyama N, Iwata T, et al. Preoperative serum oxidative stress marker as a strong indicator of nodal involvement in clinical stage I lung adenocarcinoma. Int J Clin Oncol 2012; 17: 250-5.

4) Miyazaki T, Takagi K, Mine M, et al. Video-assisted thoracic surgery attenuates perioperative oxidative stress response in lung cancer patients: a preliminary study. Acta Med Nagasaki 2014; 59: 19-25.

5) Gencer M, Ceylan E, Aksoy N, et al. Association of serum reactive oxygen metabolite levels with different histopathological types of lung cancer. Respiration 2006; 73: 520-4.

6) Trotti R, Carratelli M, Barbieri M. Performance and clinical application of a new, fast method for the detection of hydroperoxides in serum. Panminerva Med 2002; 44: 37-40.

7) Miyazaki T, Yamasaki N, Tsuchiya T, et al. Infectious episodes lead to the oxidative stress response after lung transplantation. Am J Case Rep 2015; 16: 255-8.

8) McMillan DC. The systemic inflammation-based Glasgow prognostic score: a decade of experience in patients with cancer. Cancer Treat Rev 2013; 39: 534-40.

9) Forrest LM, McMillan DC, McArdle CS, et al. Evaluation of cumulative prognostic scores based on the systemic inflammatory response in patients with inoperable non-small-cell lung cancer. Br J Cancer 2003; 89: 1028-30.

10) Lee Y, Kim SH, Han JY, et al. Early neutrophil-tolymphocyte ratio reduction as a surrogate marker of prognosis in never smokers with advanced lung adenocarcinoma receiving gefitinib or standard chemotherapy as first-line therapy. J Cancer Res Clin Oncol 2012; 138: 2009-16.

11) Tomita M, Shimizu T, Ayabe T, et al. Preoperative neutrophil to lymphocyte ratio as a prognostic predictor after curative resection for non-small cell lung cancer. Anticancer Res 2011; 31: 2995-8.

12) Warwick R, Mediratta N, Shackcloth M, et al. Preoperative red cell distribution width in patients undergoing pulmonary resections for non-small-cell lung cancer. Eur J Cardiothorac Surg 2014; 45: 108-13.

13) Mateu-Jimenez M, Sanchez-Font A, Rodriguez-Fuster A, et al. Redox balance in lung cancer patients with underlying chronic respiratory conditions. Mol Med 2016; 22: 85-98.

14) Tsukioka T, Nishiyama $N$, Iwata $T$, et al. Increase in preoperative serum reactive oxygen metabolite levels indicates nodal extension in patients with clinical stage I lung adenocarcinoma. Gen Thorac Cardiovasc Surg 2011; 59: 335-40. 